\title{
Ordering phenomena of high-spin/low-spin states in stepwise spin-crossover materials described by the ANNNI model
}

\author{
Hiroshi Watanabe, ${ }^{1,2}$ Koichiro Tanaka, ${ }^{3}$ Nicolas Bréfuel, ${ }^{4}$ Hervé Cailleau, ${ }^{1}$ Jean-François Létard, ${ }^{5}$ Sylvain Ravy, ${ }^{6,7}$ \\ Pierre Fertey, ${ }^{6}$ Masamichi Nishino, ${ }^{8}$ Seiji Miyashita, $,{ }^{9}, 10$ and Eric Collet ${ }^{1, *}$ \\ ${ }^{1}$ Institut de Physique de Rennes, Université de Rennes I - Centre national de la recherche scientifique (CNRS), \\ Unité mixte de recherche (UMR) 6251, 35042 Rennes, France \\ ${ }^{2}$ Institute of Solid State Physics, The University of Tokyo, Japan \\ ${ }^{3}$ Institute for Integrated Cell-Material Sciences, Kyoto University, Sakyo-ku, Kyoto 606-8501, Japan \\ ${ }^{4}$ Observatoire des Micro et Nanotechnologies, Unité Mixte de Service CNRS-CEA n²920, 17 Rue des Martyrs, 38054 Grenoble, France \\ ${ }^{5}$ CNRS-Université de Bordeaux, ICMCB, 87 Av. du Doc. A. Schweitzer, 33608 Pessac, France \\ ${ }^{6}$ Synchrotron SOLEIL, L'Orme des Merisiers, Saint Aubin B.P. 48, 91192 Gif-sur-Yvette, France \\ ${ }^{7}$ Laboratoire de Physique des Solides, CNRS UMR 8502, Université Paris-Sud, 91405 Orsay, France \\ ${ }^{8}$ Computational Materials Science Center, National Institute for Materials Science, Tsukuba, Ibaraki 305-0047, Japan \\ ${ }^{9}$ CREST, Japan Science and Technology Agency (JST), K's Gobancho, 7 Gobancho, Chiyoda-ku, Tokyo 102-0076, Japan \\ ${ }^{10}$ Department of Physics, Graduate School of Science, The University of Tokyo, Bunkyo-Ku, Tokyo, Japan \\ (Received 17 September 2015; revised manuscript received 18 December 2015; published 13 January 2016)
}

\begin{abstract}
We study the complex spin-state switching mechanism in a bistable molecular crystal undergoing a stepwise conversion from high-spin to low-spin states at thermal equilibrium as well as during the relaxation process from the photoinduced metastable state. We experimentally evidence that such steps are associated with complex types of long-range and short-range ordering phenomena, resulting from the occupational modulation of bistable molecular magnetic states. The conversion is then described by using two order parameters: the totally symmetric average high spin fraction and the symmetry breaking ordering parameter. The use of the anisotropic next-nearestneighbor Ising (ANNNI) model allows us to describe the microscopic origin of the ordering, and Monte Carlo simulations reproduce the observed stepwise thermal phase transition as well as the stepwise relaxation from the photoinduced high-spin state to the low-spin state.
\end{abstract}

DOI: 10.1103/PhysRevB.93.014419

\section{INTRODUCTION}

The control of the functionality of materials requires understanding the complex organization of their atomic or electronic constituents but also understanding the bi-stable or multistable processes addressable by external stimuli, including light for the photocontrol. Among multistable molecular systems, spin-crossover ( $\mathrm{SCO}$ ) compounds are prototype switchable materials. The constituting $\mathrm{Fe}^{\mathrm{II}}$ based transition-metal complexes of $3 d^{6}$ electronic configuration may be switched between high-spin (HS, $S=2$ ) and low-spin (LS, $S=0$ ) states $[1,2]$. The possibility offered by various external control parameters such as temperature, pressure, or light irradiation for balancing the relative population between LS and HS states has attracted much interest over the past decades. These prototypical photoactive systems also open a large field for basic scientific research in condensed matter for understanding the microscopic processes involved in macroscopic material's response to light. In crystals, light-induced excited spin-state trapping (LIESST) can give rise to long-lived photoinduced HS (PIHS) or photoinduced LS states at low temperature [3-8] driven by weak continuous irradiation. In addition, ultrafast photoswitching abilities driven by pulsed laser were also revealed in a complex out-of-equilibrium dynamical process [9-19].

\footnotetext{
*Corresponding author: eric.collet@univ-rennes1.fr
}

From a physical point of view, these systems are very appealing because they nicely illustrate how the subtle balance between external stimulation and intramolecular and intermolecular interactions gives rise to a large variety of conversion mechanisms of the molecular states in the crystal. In addition to simple, and more or less cooperative, entropydriven macroscopic conversion from LS to HS phases, complex phenomena resulting from the partial spin conversion and the ordering of HS and LS states around stepwise transitions in an intermediate phase (IP) have been reported. Diffraction techniques evidenced different types of long-range periodic [20-38] and aperiodic [39] spin-state ordering, as well as short-range ordering [40]. Such HS-LS order was described by different models $[41,42]$, including sometimes intrasublattice "ferro" interaction and intersublattice "antiferro" interactions [43]. However, such models cannot explain the more complicated HS-HS-LS-LS order recently reported [20] in the SCO material $\left[\mathrm{Fe}^{\mathrm{II}} \mathrm{H}_{2} \mathrm{~L}^{2-\mathrm{Me}}\right]\left[\mathrm{PF}_{6}\right]_{2}$ under consideration here (where $\mathrm{H}_{2} \mathrm{~L}^{2-\mathrm{Me}}$ denotes the organic ligand bis[((2-methylimidazol4-yl)methylidene)-3-aminopropyl] ethylenediamine). The formation of a HS-HS-LS-LS state at thermal equilibrium was theoretically studied by Boukheddaden et al. with a transfer matrix method for a one-dimensional (1D) version of an axial next-nearest-neighbor Ising-like model [44]. However, three-dimensional (3D) packing, phase diagram, or stability regions could not be investigated with this $1 \mathrm{D}$ model. In the present paper, we provide a detailed experimental analysis of the two-step $\left[\mathrm{Fe}^{\mathrm{II}} \mathrm{H}_{2} \mathrm{~L}^{2-\mathrm{Me}}\right]\left[\mathrm{PF}_{6}\right]_{2}$ SCO compound, both at thermal equilibrium and during relaxation from the PIHS 
state at low temperature. We also evidence at low temperature a metastable state with HS-HS-LS-LS order. Two order parameters are needed to describe such stepwise transition: the average HS fraction $\left(\gamma_{H S}\right)$, which is a totally symmetric order parameter, and the symmetry breaking order parameter $\eta$ measuring the amplitude of the HS-HS-LS-LS order. For understanding the origin of such a two-step conversion with complex order, we use the anisotropic next-nearest-neighbor Ising model (ANNNI). The competition between ferromagneticlike and antiferromagneticlike interactions between similar bistable spin-state ions reproduces the main features of the experimental data: a high-temperature step with a second order transition from HS to HS-HS-LS-LS order, a low-temperature step with a first-order transition towards the LS state, a two-step relaxation from the photoinduced state, as well as a metastable HS-HS-LS-LS at low temperature.

Section II presents the experimental details. Section III provides the experimental investigation and description of the two-step spin-state switching at thermal equilibrium and introduces the relevant order parameters describing the evolution of the system. Section IV presents the experimental study of the two-step relaxation process from the photoinduced state at low temperature after continuous wave (cw) laser excitation. Section V is devoted to the phenomenological descriptions of the microscopic origin of the HS-HS-LS-LS order with the ANNNI model by using mean-field (MF) theory and Monte Carlo (MC) simulation for reproducing the phase diagram and the out-of-equilibrium relaxation dynamics from the PIHS state.

\section{EXPERIMENTAL}

\section{A. Photomagnetic measurements}

The magnetic-susceptibility $\left(\chi_{M}\right)$ measurement was performed using a Quantum Design Magnetic Property Measurement System (MPMS)-5S superconducting quantum interference device (SQUID) magnetometer, connected to a light source $\left(532 \mathrm{~nm}, \mathrm{cw}, 0.5 \mathrm{~mW} / \mathrm{mm}^{2}\right)$ for photoexcitation. The sample was irradiated at $10 \mathrm{~K}$ with different exposure times. The laser was then switched off, and the temperature increased at a rate of $0.3 \mathrm{~K} \mathrm{~min}^{-1}$.

\section{B. X-ray diffraction}

Structural investigations at thermal equilibrium and under continuous light irradiation were performed by x-ray diffraction on single crystals. The crystallographic data were collected both at thermal equilibrium and under light irradiation at low temperature $(\lambda=532 \mathrm{~nm})$, and the crystalline structure were published in [Ref. [20]]. The phase transitions were investigated through the evolution of the Bragg peaks and diffuse scattering. The experiments were performed at the Institut de Physique de Rennes on a four-circle Oxford Diffraction Xcalibur 3 diffractometer $\left(\mathrm{MoK}_{\alpha}\right.$ radiation) and at Synchrotron SOLEIL at the CRISTAL diffraction beamline. The single crystals were mounted in an Oxford Cryosystems nitrogen-flow cryostat for experiments above $78 \mathrm{~K}$ and in an Oxford Diffraction Helijet cryostat for measurements down to $10 \mathrm{~K}$.

\section{SHORT-RANGE AND LONG-RANGE HS-HS-LS-LS ORDER}

The ligand field theory established the relationship between the change of electronic and structural degrees of freedom occurring during SCO. $\mathrm{Fe}^{\mathrm{II}}$ complexes in a nearly octahedral ligand field are associated with a splitting of the Fe $d$ orbitals among $t_{2 g}$ and $e_{g}$-like orbitals. Such complexes are bistable as their electronic configuration can change from $t_{2 g}^{6} e_{g}^{0}$ in the LS state to $t_{2 g}^{4} e_{g}^{2}$ in the HS state [1]. The SCO phenomenon is described by the change of the fraction of HS molecules:

$$
\gamma_{H S}=N_{H S} / N
$$

where $N_{H S}$ is the number of molecules in the HS state among the $N$ molecules of the crystal. In many systems, the LS state is the ground state, whereas the HS state of higher entropy can be populated at high temperature and $\gamma_{H S}$ varies from 0 to 1 as temperature increases. Magnetic measurements can directly probe how $\gamma_{H S}$ changes through the evolution of the $\chi_{M} \times T$ product $\left(\chi_{M}\right.$ being the molar magnetic susceptibility and $T$ the temperature). $\chi_{M} \times T$ approaches $3.5 \mathrm{~cm}^{3} \mathrm{~K} \mathrm{~mol}^{-1}$ for the HS $(S=2)$ state and equals almost 0 for the diamagnetic $S=0$ state [20]. $\chi_{M} \times T$ is therefore easily scaled to $\gamma_{H S}$, which is a totally symmetric order parameter describing the SCO. As the HS state is less bonding, the change of spin state from LS to HS is accompanied by an elongation of the average distance between the $\mathrm{Fe}$ and the $6 \mathrm{~N}$ atoms bonding it to the ligand. Typical values are $\langle\mathrm{Fe}-\mathrm{N}\rangle_{L S} \approx 2.0 \AA$ and $\langle\mathrm{Fe}-\mathrm{N}\rangle_{H S} \approx$ $2.2 \AA[6,7]$. Therefore, $\mathrm{x}$-ray diffraction is also a probe directly sensitive to the spin state through the structural change coupled to the change of electronic state. The appearance of two-step transitions associated with an IP for which $\gamma_{H S}$ is close to $1 / 2$, $1 / 3,1 / 4,3 / 4$, or is irrational [20-38] results from symmetry breaking and ordering of molecules in HS and LS states and is well probed by $\mathrm{x}$-ray diffraction.

This is the case for the $\left[\mathrm{Fe}^{\mathrm{II}} \mathrm{H}_{2} \mathrm{~L}^{2-\mathrm{Me}}\right]\left[\mathrm{PF}_{6}\right]_{2}$ material investigated here. The temperature dependence of the HS fraction $\gamma_{H S}$ shown in Fig. 1, extracted from magnetic susceptibility measurements [20], indicates a two-step SCO conversion. A preliminary analysis [20] demonstrated that above $250 \mathrm{~K}$ all molecules are in the HS state $\left(\gamma_{H S}=\right.$ 1 ), with two symmetrically equivalent molecules per unitcell (one independent crystallographic site). Below $250 \mathrm{~K}$, $\gamma_{H S}$ continuously decreases and reaches a pseudoplateau in the $[97 \mathrm{~K}-142 \mathrm{~K}$ ] domain. In this IP, approximately $50 \%$ of the molecules are in LS and HS states $\left(\gamma_{H S}=0.5\right)$. The symmetry breaking in this IP was evidenced by $\mathrm{x}$-ray diffraction and corresponds to a doubling of the crystalline $c$ axis, characterized by the appearance of additional Bragg reflections [20]. In the doubled cell on the plateau, two crystallographically independent molecular sites are observed. $\mathrm{X}$-ray diffraction data at $120 \mathrm{~K}$ show that site $A$ is mainly $\mathrm{HS}$, as characterized by $\left\langle\mathrm{Fe}_{\mathrm{A}}-\mathrm{N}\right\rangle=2.145(10) \AA$, and site $B$ is mainly LS with $\left\langle\mathrm{Fe}_{\mathrm{B}}-\mathrm{N}\right\rangle=2.050(10) \AA$ (Fig. 1). The overall bond length $\langle\mathrm{Fe}-\mathrm{N}\rangle=2.09 \AA$ between sites $A$ and $B$ indicates an average half conversion toward the LS phase in agreement with magnetic susceptibility. The structure on the plateau is therefore made of stripes of HS and LS layers alternating in a HS-HS-LS-LS sequence (Fig. 2). 


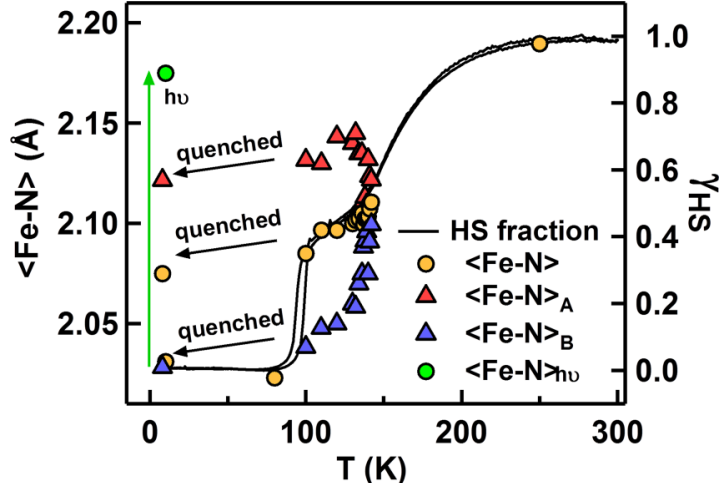

FIG. 1. Temperature dependence of $\gamma_{H S}$ (right axis) for $\left[\mathbf{F e}^{\mathrm{II}} \mathbf{H}_{2} \mathbf{L}^{2-\mathrm{Me}}\right]\left[\mathbf{P F}_{6}\right]_{2}$ determined from magnetic susceptibility measurements (continuous line) measured on cooling and warming modes. The conversion from HS $\left(\gamma_{H S}=1\right)$ to intermediate $\left(\gamma_{H S}=\right.$ $0.5)$ phases is continuous, whereas the conversion from intermediate to LS $\left(\gamma_{H S}=0\right)$ phases is discontinuous, as characterized by the appearance of the thermal hysteresis around $95 \mathrm{~K} . \gamma_{H S}$ was also determined by $\mathrm{x}$-ray diffraction through the $\langle\mathrm{Fe}-\mathrm{N}\rangle$ bond length (left axis). $\langle\mathrm{Fe}-\mathrm{N}\rangle$ is given for the molecular sites on $\mathrm{Fe}_{A}$ (red triangle) and $\mathrm{Fe}_{B}$ (blue triangle) as well as for the average over all the molecular sites (circles), which are equivalent in the HS, LS, and PIHS phases (green). Values for the quenched and photoinduced states are also indicated.

The fact that in the IP the sites are not completely HS or LS $\left(\left\langle\mathrm{Fe}_{A}-\mathrm{N}\right\rangle \neq\langle\mathrm{Fe}-\mathrm{N}\rangle_{H S}=2.190 \AA\right.$ and $\left\langle\mathrm{Fe}_{B}-\mathrm{N}\right\rangle \neq$ $\langle\mathrm{Fe}-\mathrm{N}\rangle_{L S}=2.012 \AA$ ) results from the partial spin-state ordering. This ordering can be described in the framework of the Landau theory of phase transitions [45], where the spatial HS fraction $\gamma_{H S}(r)$, equivalent to a density, in the intermediate low symmetry phase can be simply described as a spin-state concentration wave (SSCW) $[20,39,46]$. In the high symmetry HS phase, $\gamma_{H S}(r)$ is the same for all molecular sites in position $r$, which are all equivalent by applying the symmetry operators of the HS space group, especially the translation symmetry $c$ since the different molecular sites along $r / / c$ have the same

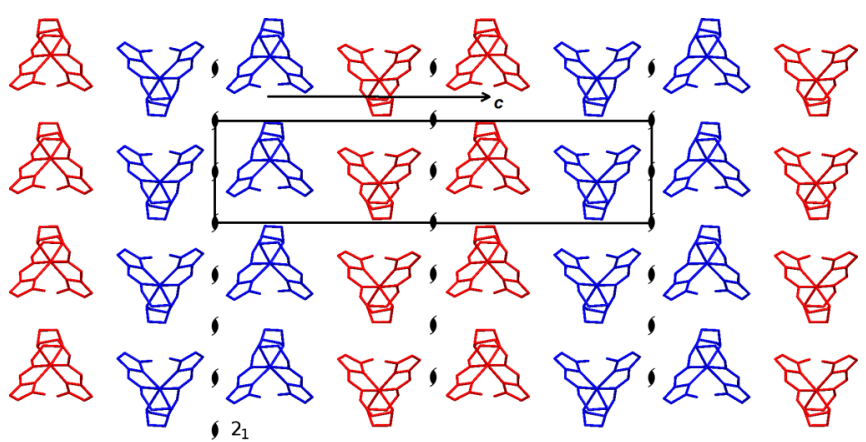

FIG. 2. Striped structure of the IP with the periodic unit cell indicated by the balck rectangle. The cell doubling (horizontal axis) is associated with the long-range order of two independent molecular sites of mainly HS (red: $A$ sites) and LS (blue: $B$ sites) population. The two LS and the two HS sites are equivalent by the $2_{1}$ screw axis, giving rise to the sequence of HS-HS-LS-LS layers along the $c$ axis. In the HS phase, the red and blue sites are equivalent by the $2_{1}$ screw axis in between them and the translation symmetry $c$.
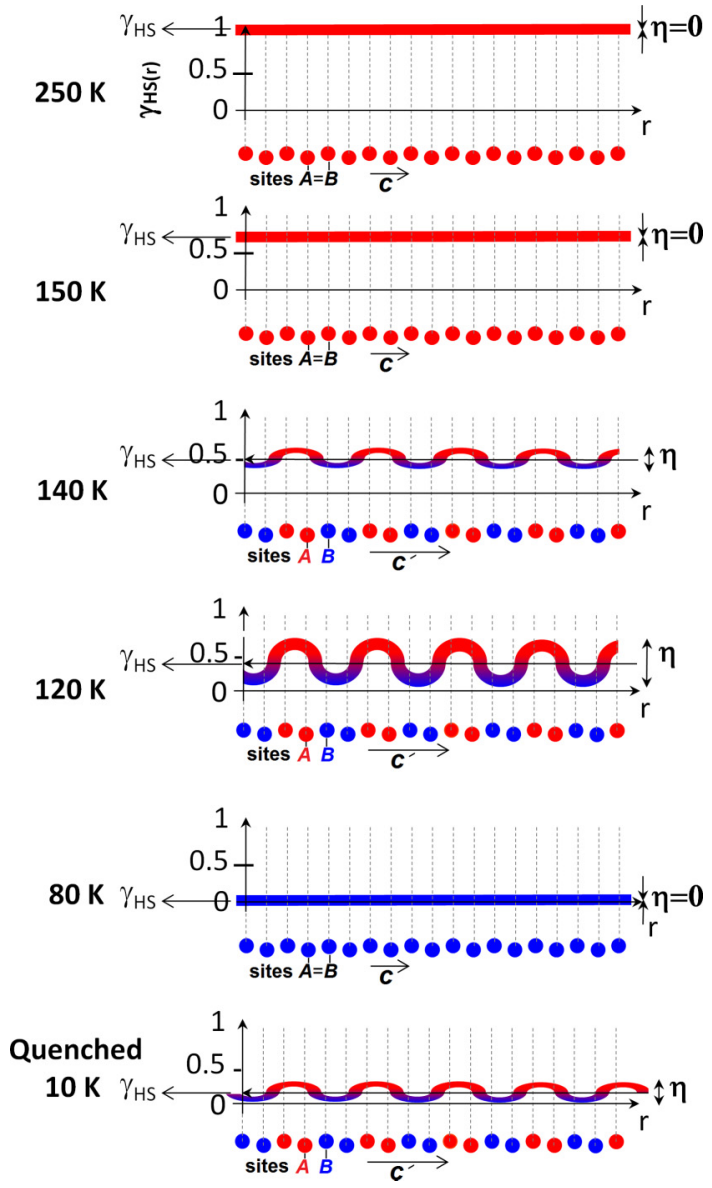

FIG. 3. Schematic representation of the spin-state conversion in terms of spin-state concentration waves among molecular sites $A$ and $B$. It is represented by $\gamma_{H S}(r)=\gamma_{\mathrm{HS}}+\eta \times \cos (q . r)$, where $q$ corresponds to the cell doubling along $c$. In the mainly HS (above $142 \mathrm{~K}$ ) and LS (below $94 \mathrm{~K}$ ), sites $A$ and $B$ are equivalent, and there is no wave. In the IP, a SSCW appears around an average value $\gamma_{H S} \approx 0.5$ with an amplitude $\eta$, which is the symmetry breaking order parameter. Both $\gamma_{H S}$ and $\eta$ depend on temperature.

probability to be in the HS state:

$$
\gamma_{H S}(\boldsymbol{r})=\gamma_{H S}(\boldsymbol{r}+n \boldsymbol{c})=\gamma_{H S}, n \in \mid \mathrm{N} .
$$

Figure 3 shows examples where $\gamma_{H S}(\boldsymbol{r})=1$ corresponds to a pure HS state above $200 \mathrm{~K}$ and $\gamma_{H S}(\boldsymbol{r})=0.6$ corresponds to a mainly HS state around $150 \mathrm{~K}$.

Around $142 \mathrm{~K}$, a symmetry breaking occurs, corresponding to a cell doubling along the $c$ axis [20], with a new periodicity $c^{\prime}=2 c$. Therefore, two distinct molecular sites around $\mathrm{Fe}_{A}$ and $\mathrm{Fe}_{B}$ appear, and a SSCW forms as

$$
\begin{aligned}
\gamma_{H S}(\boldsymbol{r}) & =\gamma_{H S}\left(\boldsymbol{r}+\boldsymbol{c}^{\prime}\right) \\
& =\gamma_{H S}(\boldsymbol{r}+2 n \boldsymbol{c}) \neq \gamma_{H S}(\boldsymbol{r}+(2 n+1) \boldsymbol{c}) .
\end{aligned}
$$

The IP can then be simply described as a spatial modulation of the probability for a site in position $r$ to be in the HS state, which can be expressed in the simple way as a sinusoidal modulation:

$$
\gamma_{H S}(\boldsymbol{r})=\gamma_{H S}+\eta \times \cos (\boldsymbol{q} \cdot \boldsymbol{r}),
$$




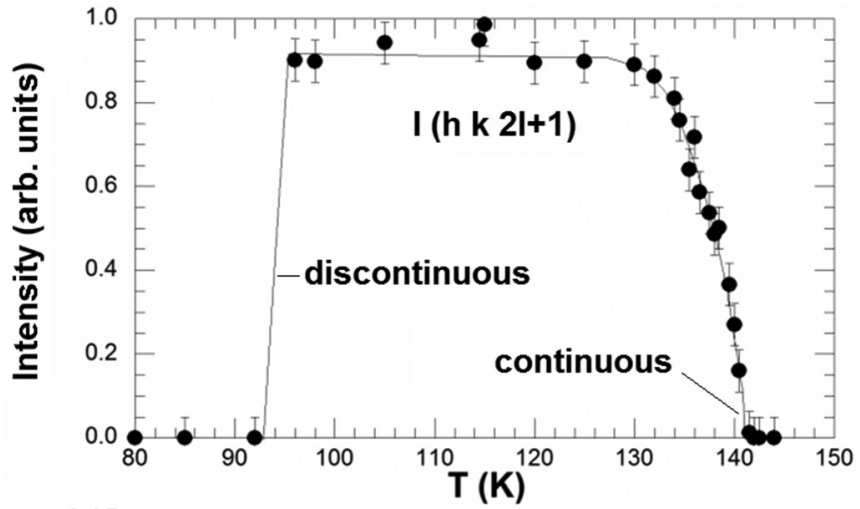

FIG. 4. Temperature dependence of the diffracted intensity for the Bragg peaks indexed $(h k 2 l+1)$ in the doubled cell.

where $\gamma_{H S}$ is the average HS fraction at a given temperature $\left(\gamma_{H S} \approx 0.5\right.$ on the plateau), $q$ is the wave vector corresponding to a cell doubling along the $c$ axis $\left(q=c^{*} / 2=c^{\prime *}\right)$, and the order parameter $\eta$ measures the amplitude of the wave. Since there are two equivalent ions in the high symmetry cell, the cell doubling gives rise to two adjacent sites in a mainly HS state and two adjacent sites in a mainly LS state, as schematically represented in Fig. 3. The intensity $I(h k 2 l+1)$ of the Bragg peaks characterizing the cell doubling, indexed $(h k 2 l+1)$ with $l$ integer in the cell $c^{\prime}=2 c$, is proportional to $\eta^{2}$ [46]. The temperature dependence of $I(h k 2 p+1)$ shown in Fig. 4 indicates a continuous phase transition for the high temperature step of the plateau and a first-order phase transition for the low temperature step of the plateau, in agreement with magnetic data.

The temperature dependence of magnetic susceptibility measurements is directly related to the evolution of the concentration of HS species, i.e., $\gamma_{H S}$. But the determination of $\eta$ requires using probes sensitive to the symmetry breaking and to the presence of two distinct molecular sites around $\mathrm{Fe}_{A}$ and $\mathrm{Fe}_{B}$. The $\langle\mathrm{Fe}-\mathrm{N}\rangle$ bond is a well-known marker of the HS fraction [47] as $\gamma_{H S}$ weights the contribution of HS and LS molecules in the observed value of $\langle\mathrm{Fe}-\mathrm{N}\rangle$. In the HS state, $\langle\mathrm{Fe}-\mathrm{N}\rangle_{H S}=2.190 \AA$, and in the LS state $\langle\mathrm{Fe}-\mathrm{N}\rangle_{L S}=2.012 \AA$ (Fig. 1). We therefore deduce for the independent molecular sites $A$ and $B$ in the IP the local HS fractions $\gamma_{H S A}$ and $\gamma_{H S B}$ :

$$
\gamma_{H S i}=\frac{\left\langle\mathrm{Fe}_{i}-\mathrm{N}\right\rangle-\langle\mathrm{Fe}-\mathrm{N}\rangle_{L S}}{\langle\mathrm{Fe}-\mathrm{N}\rangle_{H S}-\langle\mathrm{Fe}-\mathrm{N}\rangle_{L S}} .
$$

In this way, one can estimate from Fig. 1 that at 140 $\mathrm{K} \gamma_{H S A} \approx 0.65$ and $\gamma_{H S B} \approx 0.35$, whereas at $120 \mathrm{~K} \gamma_{H S A} \approx$ 0.70 and $\gamma_{H S B} \approx 0.2$ (Fig. 1). The SSCW therefore corresponds:

$$
\begin{aligned}
& \text { at } 140 \mathrm{~K} \text { to } \gamma_{H S}(\boldsymbol{r})=0.5+0.15 \times \cos (\boldsymbol{q} . \boldsymbol{r}) \\
& \text { at } 120 \mathrm{~K} \text { to } \gamma_{H S}(\boldsymbol{r})=0.45+0.25 \times \cos (\boldsymbol{q} . \boldsymbol{r}),
\end{aligned}
$$

as schematically shown in Fig. 3.

As shown in Fig. 1, both $\left\langle\mathrm{Fe}_{\mathrm{A}}-N\right\rangle$ and $\left\langle\mathrm{Fe}_{B}-\mathrm{N}\right\rangle$ evolve with temperature; however, above $142 \mathrm{~K}$ and below $92 \mathrm{~K}$ the two sites are equivalent, and the symmetry imposes $\gamma_{H S A}=\gamma_{H S B}$. Just above $\approx 142 \mathrm{~K}$, where $\gamma_{H S}$ remains close to 0.5 , the

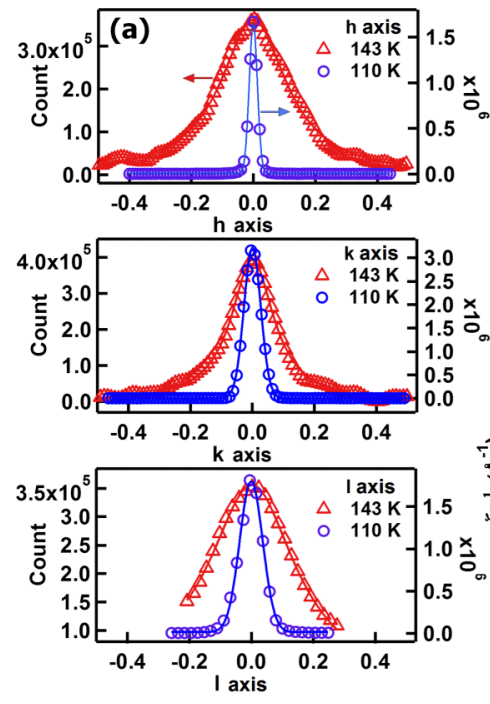

(b)
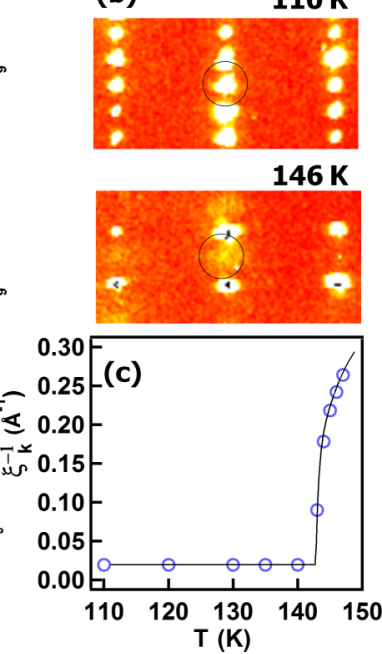

FIG. 5. (a) Scans of the diffracted intensity around superstructure Bragg peaks $(110 \mathrm{~K}$, right axis) and diffuse scattering $(143 \mathrm{~K}$, left axis) along the $h, k$, and $l$ axis and (b) in the reciprocal space. (c) This local HS-HS-LS-LS order is associated with a broadening of the peaks characterized by the evolution of $\xi_{k}^{-1}$ above $142 \mathrm{~K}$.

superlattice reflections at $c^{*} / 2$ characteristic of the SSCW continuously disappear. However, diffuse scattering around this $Z$ point of the Brillouin zone is observed (Fig. 5). Below $142 \mathrm{~K}$, the sharp superstructure peaks, as sharp as other Bragg peaks, indicate a long-range HS-HS-LS-LS order. Above $142 \mathrm{~K}$, the broad diffuse scattering around the superstructure position is reminiscent of the spatial fluctuation of the symmetry breaking order parameter, and it is associated with a short-range HS-HS-LS-LS order. Contrary to what is observed in quasi-1D systems $[40,48]$ where the diffuse scattering is located on planes, the shape of the present diffuse scattering is quite isotropic here. In Fig. 5(a), we use a Lorentzian function to describe the diffuse signal along $k$,

$$
I(k)=\mathrm{I}_{0}+\mathrm{I}_{M} /\left(1+\left((k) / \xi_{k}^{-1}\right)^{2}\right),
$$

from which we extract the inverse correlation length $\xi_{k}^{-1}$ shown in Fig. 5(c). Similar fits are performed along $h$ and $l$ axes give $\xi_{h}^{-1}$ and $\xi_{l}^{-1}$. The correlation lengths present similar values along the different directions. This means that the amplitude of the interactions along the different directions are of the same order, and this information is of importance for the theoretical description.

In addition to this main HS-HS-LS-LS order at the origin of the stepwise transition in this material (for which the unit cell can be given as $(a, b, 2 c)$ with respect to the high symmetry HS state), a weak structural distortion appears in the LS phase, and the unit cell changes to $(4 a, b, c)$. It results from a small tilting of the cations in the LS state [20]. In the PIHS state, all of the cations are in the HS state, but another structural distortion (tilting) occurs, in which the unit cell has changed to $(a, b, 3 c)$. These weak structural distortions are not relevant for describing the HS-HS-LS-LS spin-state ordering observed here in the IP, however, the associated superstructure peaks are very useful markers of the LS and PIHS phases, which will 
help in studying the relaxation dynamics from the PIHS to the photoinduced LS phases.

\section{RELAXATION FROM PHOTOINDUCED AND QUENCHED STATES}

In SCO materials, different types of metastable states related to electronic and structural changes compete at very low temperature when the thermal energy is too weak for reaching the true ground state. This is also the case in this compound since the quenched state reached by a flash cooling from room temperature to $10 \mathrm{~K}$ corresponds to the IP characterized by $a(a, b, 2 c)$ cell. The corresponding structure at $10 \mathrm{~K}$ is similar to that of the IP: one site is mainly HS, and one is mainly LS, as shown in Fig. 1. Both x-ray diffraction and magnetic susceptibility (Fig. 6) indicate a partially HS state with $\gamma_{H S}=0.2$. This quenched IP is metastable at low temperature. It relaxes within a single step around $60 \mathrm{~K}$ (Fig. 6, blue line) towards the fully LS state.

The HS state is also metastable at low temperature [20], and it is reached by continuous laser irradiation $(532 \mathrm{~nm})$. The magnetic susceptibility (Fig. 6) and $\langle\mathrm{Fe}-\mathrm{N}\rangle$ bond length (Fig. 1) indicate a fully PIHS state. The evolution of the HS fraction from this PIHS state with $1 \mathrm{~K} \mathrm{~min}^{-1}$ thermal change is also reported (green line), and a two-step relaxation from PIHS to LS state is observed (see the inset of Fig. 6), characterized by two peaks at $34 \mathrm{~K}$ and $48 \mathrm{~K}$ of the derivative of the HS fraction with temperature and suggesting that the PIHS state relaxes to the LS phase via an intermediate state. This two-step relaxation from the PIHS state is thus notably different from the single-step relaxation of the quenched state. For learning more about this two-step process, additional photomagnetic measurements were performed. Figure 7 shows the relaxation process of the PIHS state, with different exposure time (from 1 hour to 21 hours) to the same laser excitation. The temperature derivative of HS fraction shows three relaxations steps, characterized by peaks around 37,48 , and $63 \mathrm{~K}$. The amplitude of the peak around $48 \mathrm{~K}$ decreases for longer exposure time, whereas the one with the $63 \mathrm{~K}$ peak appears

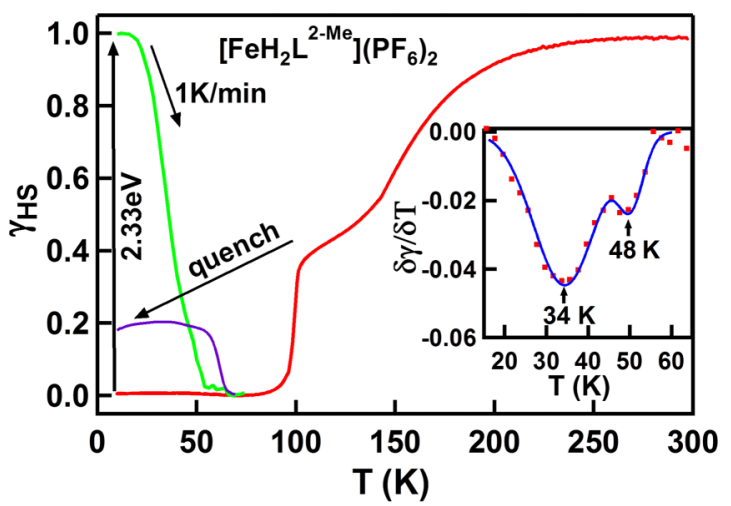

FIG. 6. Temperature dependence of HS fraction estimated by magnetic susceptibility measurements. The red line shows thermal transition from LS state, the blue line shows relaxation from quenched state, and the green line shows relaxation from PIHS state. Heating rates are $1 \mathrm{~K} \mathrm{~min}^{-1}$. The inset shows the temperature derivative of HS fraction (green line), pointing two $T_{\text {LIESST }}$ steps around $35 \mathrm{~K}$ and $50 \mathrm{~K}$.
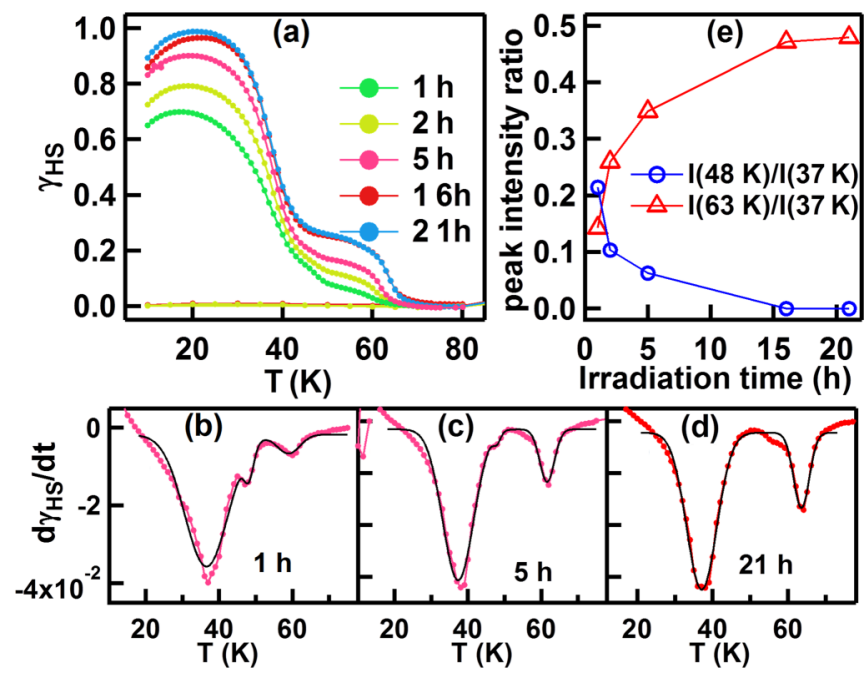

FIG. 7. (a) Temperature dependence of HS fraction estimated by magnetic susceptibility measurements after $1-21 \mathrm{~h}$ laser irradiation at $10 \mathrm{~K}$. The temperature derivative of HS fraction after (b) $1 \mathrm{~h}$, (c) $5 \mathrm{~h}$, and (d) $21 \mathrm{~h}$ laser irradiation showing relaxation peaks around $37 \mathrm{~K}$, $48 \mathrm{~K}$, and $63 \mathrm{~K}$. (e) The temperature dependence with laser exposure time of the peak intensity ratio at $48 \mathrm{~K}$ and $63 \mathrm{~K}$ with respect to $37 \mathrm{~K}$.

more and more clearly. The response is maximized after about 15 hours of laser exposure. A better characterization of these relaxations is achieved by $\mathrm{x}$-ray diffraction studies, allowing to monitor the PIHS and LS phases (Fig. 8). The crystals were also excited at $10 \mathrm{~K}$ for 15 hours. At $10 \mathrm{~K}$, the superstructure peaks at $c^{*} / 3$ characterize the PIHS phase with the $(a b 3 c)$ lattice [see also Fig. 8(c)]. Around $40 \mathrm{~K}$, these superstructure peaks disappear and an average $(a, b, c)$ cell is observed: given the limited the accuracy of the temperature control in this measurement (few K), this corresponds to the first step of the relaxation observed in Fig. 6. Around $50 \mathrm{~K}$, new Bragg peaks characteristic of the $\operatorname{LS}(4 a, b, c)$ cell appear, evidencing the second step of the relaxation.

In summary of the experimental part, three main features should be underlined. In the IP, the HS-HS-LS-LS order appears continuously around $142 \mathrm{~K}$ and disappears discontinuously around $90 \mathrm{~K}$. The relaxation from the PIHS state occurs in two steps, but in the intermediate step the Bragg
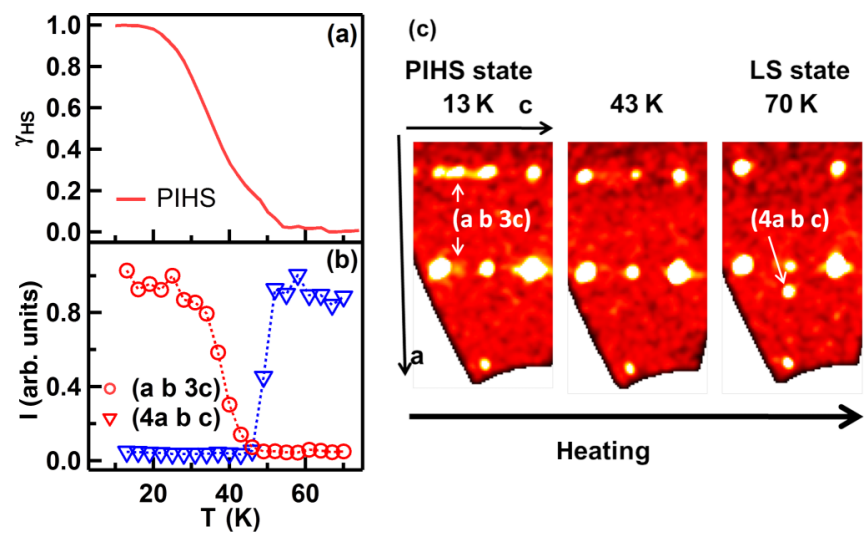

FIG. 8. (a) Temperature dependence of HS fraction and of the (b) intensity of the Bragg peaks characteristic of the PIHS $(a, b, 3 c)$ and LS $(4 a, b, c)$ cells and (c) during the relaxation from PIHS state. 
peaks characteristic of the cell doubling resulting from the HS-HS-LS-LS order are not observed. Finally, the IP with HSHS-LS-LS order can be thermally quenched in a metastable state at $10 \mathrm{~K}$.

In the following, we make use of the ANNNI model to explain the main experimental observations.

\section{THEORETICAL STUDIES WITH A MODIFIED ANNNI MODEL}

\section{A. A MF study of a modified ANNNI model}

The SCO system has a bistable nature with HS and LS states, and it has been intensively modeled by the Ising-like model [49-54] with a variable $\sigma_{i}$, which expresses the HS or LS state of the $\mathrm{i}^{\text {th }}$ molecule by $\sigma_{i}=1$ and -1 , respectively. These states are denoted + and - hereafter. Depending on the interaction among the Ising spins, the model exhibits various types of phase transitions. For example, antiferromagneticlike interaction in a bipartite system brings an IP between the HS phase at high temperatures and the LS phase at low temperatures. In the IP, a staggered spin structure appears, and the temperature dependence of the high spin fraction $\gamma_{H S}$ has a step [43,55-58 and references therin]. It is well known that the competition among the interactions (frustration) can cause various types of ordering structures that have a large unit cell compared to the high symmetry phase. Consequently, the subtle balance between the competing interactions and entropy can generate an interesting sequence of phase transitions at thermal equilibrium [59]. The present material has a layered structure (the molecules in a layer have the same spin state), and it shows HS-HS-LS-LS alternation in the IP [Figs. 2 and 9 (right)].

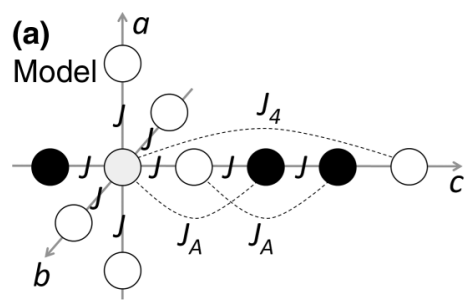

(b) Crystalline structure

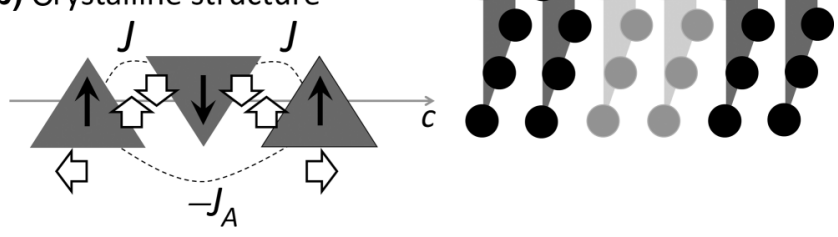

FIG. 9. (a) For the ANNNI scheme, the interaction between nearest neighbors is ferromagnetic $(J)$ in the $(a, b)$ plane and along direction $c$. The model has an antiferromagnetic interaction $\left(J_{\mathrm{A}}\right)$ along direction $c$ for the next-nearest neighbors. (b) We found two possible origins of the ferro $(J)$ and antiferro $\left(J_{\mathrm{A}}\right)$ coupling; the molecular motions (white arrows) show the decreasing of distance between nearest neighbors and increasing of distance between next-nearest neighbors, and/or the interaction between the molecular dipole moments (black arrows) of opposite sign between nearest neighbors and next-nearest neighbors. (c) The striped HS-HS-LS-LS structure, schematically represented with LS (black balls) and HS (gray balls) sites.
Such a structure is similar to the ones described in the ANNNI model [60,61].

The Hamiltonian of the ANNNI model is given by

$$
\mathcal{H}_{\mathrm{ANNNI}}=-J \sum_{\langle i j\rangle} \sigma_{i} \sigma_{j}+J_{\mathrm{A}} \sum_{\langle i k\rangle} \sigma_{i} \sigma_{k}-H \sum_{i} \sigma_{i},
$$

where $\langle i j\rangle$ denotes all the nearest neighbor pairs in the simple cubic lattice and $\langle i k\rangle$ denotes the next-nearest neighbor pairs along the $c$ direction of the lattice, following the assignment in the experimental part of the present paper [Fig. 9(a)]. It is known that because of the frustration due to the competition between the ferrolike $J$ and the antiferrolike $-J_{\mathrm{A}}$ interactions, the ground state of this model for the case $J_{\mathrm{A}} / J>1 / 2$ is the $(++--)$ state $[60,61]$. Thus, we suppose that the ANNNI model is a good candidate to describe the ordering structure of the present material.

In the present paper, we can mention structural features that can provide some grounds for a microscopic explanation of the frustrated interactions. In the crystalline structure (see [Ref. [20]] and Fig. 2), the molecules with a triangular shape alternate head-to-tail along the $c$ crystalline axis. Therefore, the interactions can qualitatively differ from nearest neighbors to next-nearest neighbors. This gives two possible origins of the ferro $(J)$ and antiferro $\left(J_{\mathrm{A}}\right)$ coupling, as indicated in Fig. 9(b). On the one hand, the molecular ions carry a dipole moment oriented along the molecular symmetry axis, as illustrated by the black arrows, and because of the molecular packing the dipolar interaction between the nearest neighbors is opposite to the one between the next-nearest neighbors. On the other hand, the sizes of HS and LS molecules are different; this fact causes elastic interaction among them, on which various interesting properties have been reported (see Refs. [51-54] and references therein). When a molecule moves to get closer to its nearest neighbors [20] [represented by vertical arrow in Fig. 9(b)], the system gains in elastic energy. But because of steric effects, the next-nearest neighbors move in the opposite direction (horizontal arrows) when the nearest neighbors get closer, and this costs in elastic energy. These observations provide a microscopic explanation of the opposite signs of $J$ and $J_{\mathrm{A}}$, even though other ones may also be involved. But the transcription of such interactions in terms of ferro and antiferro is subtle and requires future $a b$ initio calculations. In addition, it is also known that the ANNNI model has a very rich phase diagram with structures of long periods, which is called the devil's flour [60,61]. Thus, if one decreases the temperature from the disordered high temperature phase where HS and LS molecules fluctuate in time and space, the $(++--)$ structure does not appear immediately after the disordered phase. However, in the experiment, the present material only shows the $(++--)$ structure. We thus modify the ANNNI model to suppress the structures of longer periods by introducing a fourth neighbor interaction $J_{4}$, of ferromagneticlike nature, stabilizing the $(++--)$ structure,

$$
\begin{aligned}
\mathcal{H}_{\text {Modified ANNNI }}= & -J \sum_{\langle i j\rangle} \sigma_{i} \sigma_{j}+J_{\mathrm{A}} \sum_{\langle i k\rangle} \sigma_{i} \sigma_{k} \\
& -J_{4} \sum_{\langle i m\rangle} \sigma_{i} \sigma_{m}-H \sum_{i} \sigma_{i} .
\end{aligned}
$$


We found that this extended ANNNI model with $J_{\mathrm{A}} / J=$ 1.6 and $J_{4} / J=0.2$ reproduces well the experimentally found SCO behavior. In general, various parameter sets are possible. Indeed, in derivatives of this $\mathrm{SCO}$ material (using $\mathrm{AsF}_{6}^{-}$or $\mathrm{SbF}_{6}^{-}$as a counter anion instead of $\mathrm{PF}_{6}^{-}$), other types of longrange HS/LS intermediate structures of commensurate [21] and incommensurate [39] natures were also reported. In the present paper, we concentrate on the above case observed in the present $\mathrm{PF}_{6}^{-}$derivative. As it has been pointed out, the entropy driven nature of the SCO material is taken into account in the form of the temperature dependent external field $H$ in the Ising model description. Indeed, in SCO materials, the HS and LS states have different enthalpies, $h_{H S}$ and $h_{L S}$, and also different degeneracies, $g_{H S}$ and $g_{L S}$, due to the difference in the number of states in spin and different density of states of lattice vibration, etc.

With the enthalpy difference between HS and LS states,

$$
2 D=h_{\mathrm{HS}}-h_{\mathrm{LS}},
$$

and the difference of degeneracy that is given in the form of entropy difference,

$$
\Delta S=k_{B} \log \left(\frac{g_{\mathrm{HS}}}{g_{\mathrm{LS}}}\right),
$$

the effective field is given by

$$
H=-D+\left(\frac{1}{2}\right) T \Delta S=-D+\left(\frac{1}{2}\right) T k_{B} \log \left(\frac{g_{\mathrm{HS}}}{g_{\mathrm{LS}}}\right) .
$$

Thus, the SCO of this system occurs along the line $(T,-D$ $+T \Delta S / 2)$ of the phase diagram $(T, H)$ of the corresponding Ising model.

The layers have a ferromagneticlike order in the $(a, b)$ plane (perpendicular to the $c$ axis) because the molecules in a same layer are in a single + or - state. We thus describe the different phases by the sequence of spin state along the $c$ axis for the four layers: IP $(++--)$ for the IP, HS $(++++)$ for the high-spin phase, and LS (- - - ) for the low-spin phase.

Let us first study the properties at $T=0$. For a large value of $H$, the ground state is an induced ferromagneticlike state with all the molecules in the HS state $(++++)$. The energies of $(++++)$ and $(++--)$ structures are $E_{(++++)} \approx\left(-3 J+J_{\mathrm{A}}-H-J_{4}\right) N$ and $E_{(++--)} \approx(-2 J-$ $\left.J_{A}-J_{4}\right) N$, respectively.

Because $E_{(++++)}-E_{(++--)}=\left(-J+2 J_{A}-H\right) N$, the ground state changes from ferromagnetic state to staggered state at $H_{\mathrm{C}}=2 J_{\mathrm{A}}-J$. The condition to have a ferromagnetic ground state at $H=0$ is $J_{\mathrm{A}}<J / 2$. Here, we are interested in the case in which the ground state at $H=0$ is $(++--)$. Thus, we consider the following the cases in which $J_{\mathrm{A}}>J / 2$.

The point at which the energies of the ferromagnetic state and the staggered state cross is located at $H_{\mathrm{C}}=2 J_{\mathbf{A}}-J$, and it corresponds to a first order phase transition. Now we study the limit of the metastability for a single flip, and for this purpose we study the local stability of the $(++++)$ states. In this ferromagnetic state, the local field for each molecule is

$$
H_{\mathrm{f}}=6 J-2 J_{\mathrm{A}}+H+2 J_{4} .
$$

If $H_{\mathrm{f}}>0$, the $(++++)$ phase is locally stable. The critical $H_{\text {fcp }}$ field at which the $(++++)$ state becomes unstable is given by

$$
H_{\mathrm{fcp}}=2 J_{\mathrm{A}}-6 J-2 J_{4} .
$$

Similarly, the critical $H_{\mathrm{fcm}}$ field at which the $\left(---_{-}\right)$ state becomes unstable is given by

$$
H_{\mathrm{fcm}}=-H_{\mathrm{fcp}}=-2 J_{\mathrm{A}}+6 J+2 J_{4} .
$$

We also obtain the locally stable region of the $(++--)$ state. With the same of type argument as the ones above, the $(++--)$ state is stable within the region

$$
\begin{aligned}
-H_{\mathrm{ac}} & =-\left(4 J+2 J_{\mathrm{A}}+2 J_{4}\right)<H<\left(4 J+2 J_{\mathrm{A}}+2 J_{4}\right) \\
& =H_{\mathrm{ac}} .
\end{aligned}
$$

We denote the magnetizations on the four layers as $\left(m_{\mathrm{A}}, m_{\mathrm{A}}, m_{\mathrm{B}}, m_{\mathrm{B}}\right)$. Here, $m_{\mathrm{A}}$ and $m_{\mathrm{B}}$ are related to the HS fraction on each layer $\gamma_{H S A}$ and $\gamma_{H S B}$ as

$$
\gamma_{H S A}=\left(m_{A}+1\right) / 2 \text { and } \gamma_{H S B}=\left(m_{B}+1\right) / 2 \text {. }
$$

To study properties at finite temperatures, we study the model by a MF approach and MC simulation. The free energy in the MF approximation is given by

$$
\begin{gathered}
F\left(m_{A}, m_{B}\right)=E\left(m_{A}, m_{B}\right)-k_{B} T S\left(m_{A}, m_{B}\right) \\
E\left(m_{A}, m_{B}\right)=-\frac{1}{2}\left(5 J+2 J_{4}\right) m_{A}^{2}-\left(J-2 J_{A}\right) m_{A} m_{B} \\
-\frac{1}{2}\left(5 J+2 J_{4}\right) m_{B}^{2}-H\left(m_{A}+m_{B}\right) \\
S\left(m_{A}, m_{B}\right)=-\left(\frac{1+m_{A}}{2} \ln \left(\frac{1+m_{A}}{2}\right)+\frac{1-m_{A}}{2}\right. \\
\left.\times \ln \left(\frac{1-m_{A}}{2}\right)\right)-\left(\frac{1+m_{B}}{2} \ln \left(\frac{1+m_{B}}{2}\right)\right. \\
\left.+\frac{1-m_{B}}{2} \ln \left(\frac{1-m_{B}}{2}\right)\right),
\end{gathered}
$$

and the self-consistent equation is given by

$$
\begin{aligned}
m_{A}= & \tanh \left[\beta \left\{J\left(5\left\langle m_{A}\right\rangle+\left\langle m_{B}\right\rangle\right)\right.\right. \\
& \left.\left.-2 J_{A}\left\langle m_{B}\right\rangle+2 J_{4}\left\langle m_{A}\right\rangle+H\right\}\right] \\
m_{B}= & \tanh \left[\beta \left\{J\left(5\left\langle m_{B}\right\rangle+\left\langle m_{A}\right\rangle\right)\right.\right. \\
& \left.\left.-2 J_{A}\left\langle m_{A}\right\rangle+2 J_{4}\left\langle m_{B}\right\rangle+H\right\}\right] .
\end{aligned}
$$

For the present system, we use the parameters $J_{\mathrm{A}} / J=1.6$ and $J_{4} / J=0.2$, which reproduces the main experimental features and generates the phase diagram shown in Fig. 10 (energies are given in $J$ scale, with $J=1$ ). We found that the set of parameters $\left(D=6, \Delta S / k_{\mathrm{B}}=12 / 7\right)$, which corresponds to the thermodynamical path indicated by the solid green line in Fig. 10, reproduces the temperature dependence of the HS fraction as the phase transition from the disordered to the IP crosses the continuous transition line, whereas the phase transition from the IP to the LS phase crosses the discontinuous transition line. This is better seen in Fig. 11 where we plot the temperature dependence of $m_{\mathrm{A}}$ and $m_{\mathrm{B}}$ and the average magnetization of the lattice $m=\left(m_{A}+m_{B}\right) / 2$, related to $\gamma_{H S}$ by $\gamma_{H S}=(m+1) / 2$. The black squares denote the order parameter $\eta=\left|m_{\mathrm{A}}-m_{\mathrm{B}}\right| / 2$. The ferromagnetic solutions $\left(m_{\mathrm{A}}=m_{\mathrm{B}}\right)$ are plotted by black bullets. Here, we see 


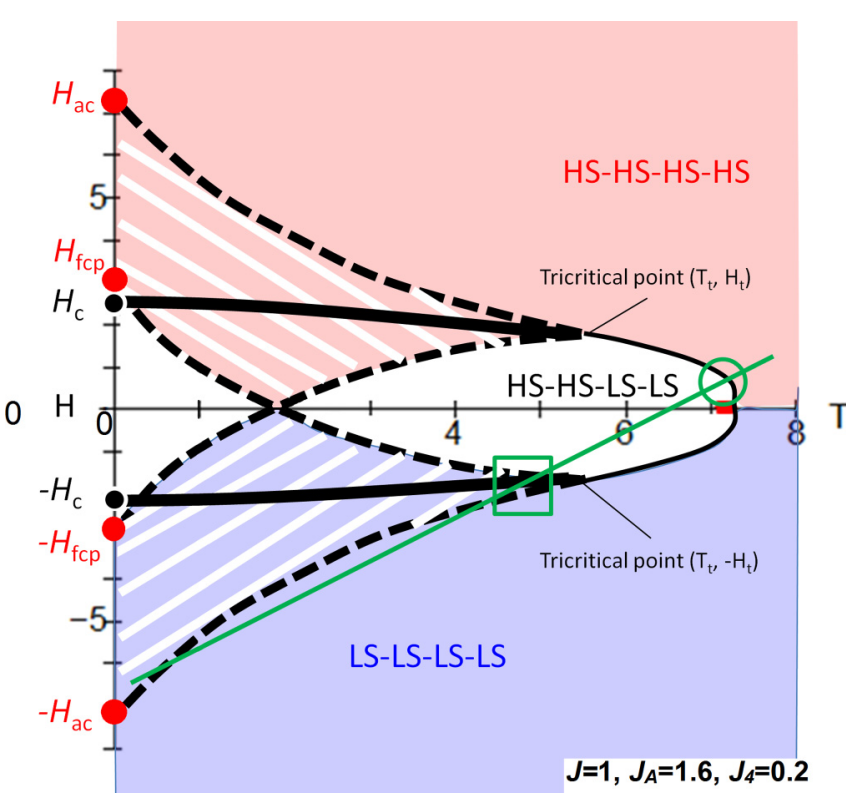

FIG. 10. Phase diagram showing the regions of $(++++)$ HS (red), $(++--)$ IP (white), and (- - - ) LS (blue) phases. There are regions in which metastable phases exist. The red dots $\left(H_{\mathrm{ac}}\right.$ and $-H_{\text {ac }}$ ) on the line of $T=0$ denote the limits of metastability of the order $(++--)$. The red dots $\left(H_{\mathrm{fcp}}\right.$ and $\left.H_{\mathrm{fcm}}=-H_{\mathrm{fcp}}\right)$ on the line of $T=0$ denote the points of end of metastability of $(++++)$ and $(++--)$, respectively. The continuous phase transition is indicated by the thin continuous line. The first-order phase transition points are indicated by thick continuous lines. During the thermal pathway shown by the green line, the phase transition between $(++++)$ and $(++--)$ (green circle) occurs at a temperature above the $\left(T_{1}, H_{1}\right)$ tricritical point; this phase transition is of second order. The phase transition between $(++--)$ and $(----)$ (green square) occurs at a temperature below the $\left(T_{1},-H_{1}\right)$ tricritical point below; this phase transition is of first-order. In the shaded areas with downward (upward) strip, both the IP and HS (LS) phases are locally stable. The $(++--)$ state is metastable in the region surrounded by curves $\left[H_{\mathrm{C}}-\right.$ the upper tricritical point $\left.-H_{\mathrm{ac}}\right]$. The $(++++)$ state is metastable in the region $\left[H_{\mathrm{C}}-\right.$ the upper tricritical point $\left.-H_{\mathrm{fcp}}\right]$. The same structure exists for $(++--)$ and $(----)$ as a reflection by the $H=0$ line. In the white region at low temperature, the three phases of $(++++),(++--)$ and $(----)$ are locally stable.

the second-order phase transition from HS state to $(++--)$ state at $T=7.5$ and a first-order phase transition around $T=5$. The downward arrow denotes the transition from IP to LS, and the upward arrow denotes the transition from LS to IP. At low temperature, the LS phase is stable, but we also find a metastable branch of $(++--)$ structure below $T=2$. This structure gives a qualitative explanation of the experimental observation.

\section{B. MC simulations}

In the previous subsection, we study the ordering process by a MF method. The MF phase diagram gives a good qualitative description for the ordering properties of the system including the metastable state. But the MF does not include the microscopic fluctuation, and quantitatively the ordered regions are generally shrunk to the low temperature side. Thus, we

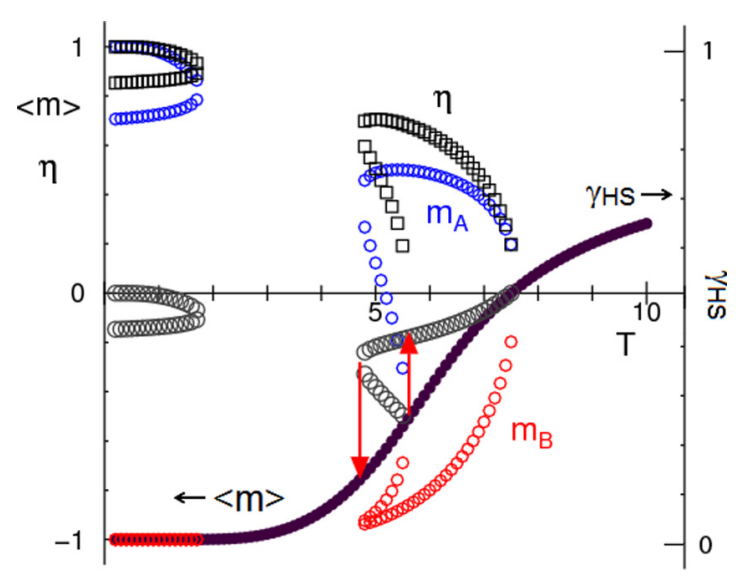

FIG. 11. Temperature dependence of the order parameters (MF analysis). The black bullets denote the ferromagnetic solution. The blue and red circles denote the staggered solution $\left(m_{\mathrm{A}}, m_{\mathrm{B}}\right)$, and large black circles denote the average total magnetization $m=$ $\left(m_{\mathrm{A}}+m_{\mathrm{B}}\right) / 2$, which correspond to $\gamma_{H S}=(m+1) / 2$ shown on the right axis. The squares denote the order parameter $\eta=\left|m_{\mathrm{A}}-m_{\mathrm{B}}\right| / 2$.

also studied the present model by a MC simulation. We found that the parameter set $\left(J_{\mathrm{A}} / J=1.6, J_{4} / J=0.2, D=8\right.$, and $g=30$, with $\left.g=g_{\text {HS }} / g_{\text {LS }}\right)$ gives a good agreement of the overall temperature dependence of the SCO component in the cooling process. We show in Fig. 12 the temperature dependence of the magnetization (and therefore of the HS fraction $\left.\gamma_{H S}\right)$ with $\gamma_{H S}=(m+1) / 2$, the correlation $(C)$ of the second nearest neighbor in the $c$ axis,

$$
C=\left\langle\sigma_{i, j, k} \sigma_{i, j, k+2}\right\rangle
$$

and the order parameter

$$
\eta=\frac{1}{N} \sqrt{\left\langle\left(\sum_{i, j, k=1}^{N} \sigma_{i, j, k}\left[\cos \left(\frac{2 \pi}{4} k\right)-\sin \left(\frac{2 \pi}{4} k\right)\right]\right)^{2}\right\rangle}
$$

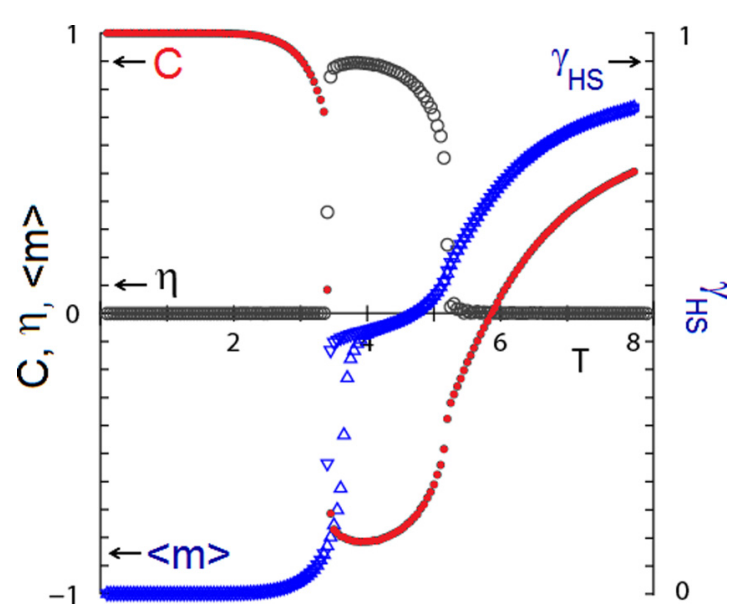

FIG. 12. Temperature dependences of the magnetization (triangles), which correspond to $\gamma_{H S}$ (right axis), the order parameters $\eta$ (open circles), and the correlation function $C$ (closed circles) (MC simulations). 
equal to 1 when the spin-state order along $k$ corresponds to a complete order, with the sequence of $\sigma_{i, j, k}(++--)$. However. since an antiphase long-range domain may form $(-++-), \eta$ is defined in the MC by

$$
\begin{gathered}
\eta=\max \left(\frac{1}{N} \sqrt{\left.\left\langle\sum_{i, j, k=1}^{N} \sigma_{i, j, k}\left[\cos \left(\frac{2 \pi}{4} k\right)+\sin \left(\frac{2 \pi}{4} k\right)\right]\right)^{2}\right\rangle_{M C}},\right. \\
\frac{1}{N} \sqrt{\left.\left\langle\left(\sum_{i, j, k=1}^{N} \sigma_{i, j, k}\left[\cos \left(\frac{2 \pi}{4} k\right)-\sin \left(\frac{2 \pi}{4} k\right)\right]\right)^{2}\right\rangle_{M C}\right)} .
\end{gathered}
$$

In Fig. 12, we find that the symmetry breaking order parameter $\eta$ develops in the IP. The correlation $C$ is negative in the IP, where along the $c$ crystalline axis molecules on sites $k$ and $k+2$ are of the opposite spin state $\left(\sigma_{k}=-\sigma_{k+2}\right)$ reflecting the order parameter. We also find that $C$ begins to develop at higher temperatures than $T_{C}$ where the short range $(++--)$ cluster ordering appears. There exists a hysteresis at the low temperature end of the IP. We also depict spatial configurations of LS and HS states at characteristic temperatures in Fig. 13. Above $T=6$, there are few molecules in the LS state. Just above the continuous phase transition of intermediate plateau $(T=5.2)$, we find the local stripe structure $(++--)$ indicated by boxes in Fig. 13(c). This observation corresponds to the diffuse scattering observed in Fig. 5 at $143 \mathrm{~K}$ and $146 \mathrm{~K}$.

We now discuss the relaxations from PIHS state and quenched $(++--)$ state. In experiments, we found that during the relaxations from the PIHS state, an intermediate step appears (with a fraction of molecules in the HS state). We also observed a quenched $(++--)$ structure, which stays rather stable up to $60 \mathrm{~K}$. The appearance of such a metastable state indicates the presence of the metastable branch of the $(++--)$ structure in the phase diagram. In the MF analysis, we found such a metastable state at low temperatures, as seen in Fig. 10, and here we investigate such a metastable state in the MC study. However, the system with the parameter set $\left\{J_{\mathrm{A}} / J=1.6, J_{4} / J=0.2, D=8\right.$, and $\left.g=30\right\}$ does not present such a metastability. Thus we suppose that the parameter set may change in the PIHS or quenched $(++--)$ phases. Indeed, it has been pointed out that the photoinduced state has a three times large unit cell $(a, b, 3 c)$. The resulting loss of twofold symmetry on the molecule [20] splits energy levels, and the orbital degeneracy is reduced [62]; thus, we expect that the value of $D$ is reduced and also that the value of $g$ is smaller. Taking into account this characteristic, we assume that the value of the effective field $H=-D+k_{\mathrm{B}} T \ln (g) / 2$ has a small value. In simulation, we set the value of $H$ to be -2.4 at $T=0.1$. With this setting of the parameter, we find a good agreement of the relaxations, as depicted experimentally in Fig. 6. Here it should be noted that the HS state is still thermodynamically unstable even with the choice of the parameters mentioned above. Thus, we do not have the metastability for the HS phase, but because of the Arrhenius mechanism, the HS fraction remains at low temperatures. Taking these facts into account, we study the behavior of the relaxation of the HS fraction from PIHS (Fig. 14). The HS state is unstable and gradually relaxes to LS state. In the process, part of the system is trapped with the $(++--)$ configuration when $\gamma_{H S} \approx 0.5$ (point A in Fig. 14), as found in the experiment. However, this order is local, as characterized by the value of the symmetry breaking order parameter $\eta \approx$ 0 and the negative correlation $C$. Therefore, only short-range HS-HS-LS-LS order forms, but no long-range order forms during this out-of-equilibrium process, in agreement with the nonobservation of Bragg peaks (Fig. 8) characteristic of the $(++--)$ phase during the experimental study of the relaxation from the PIHS state.

When the $(++--)$ phase is quenched to a low temperature, the lattice has again a large unit $(a, b, 2 c)$, and we expect again the change of thermodynamical parameters. In this case, we set $H=-3.0$ at low temperatures. If we use the same thermodynamical parameters as for the case of the PIHS $(a, b, 3 c)$ structure, we found that the quenched $(++--)$ state is stable on a plateau at low temperature up to a higher temperature than the PIHS. In the simulation, we set the complete $(++--)$ structure at $T=0.1$. Thus, the HS fraction is $\gamma_{H S}=0.5$, and the order is complete, as characterized by $\eta=1$ and $C=-1$, up to $T \approx 2.2$ (point $\mathrm{B}$ in Fig. 14), where $\gamma_{H S}$ decreases. Above this temperature, the LS phase forms: both $\gamma_{H S}$ and $\eta$ reach 0 , whereas $C$ reaches (a) $\quad \mathrm{T}=3.4$

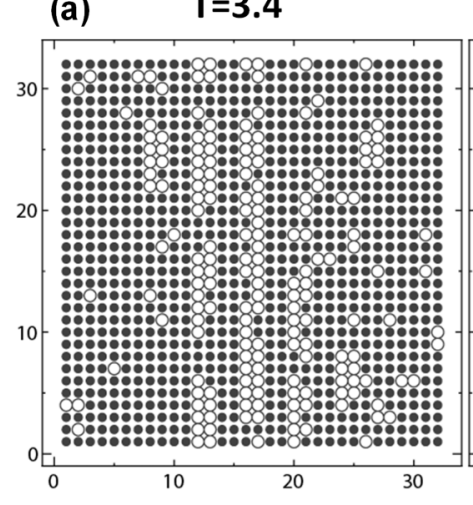

(b) $\quad \mathrm{T}=4.0$

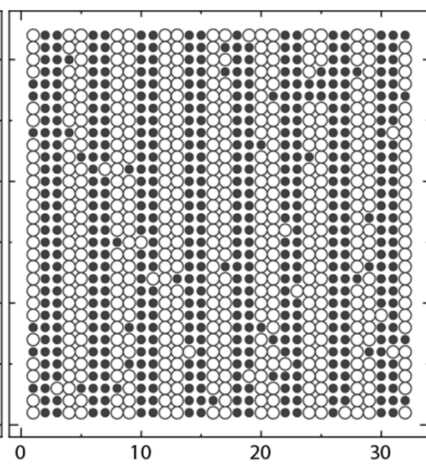

(c) $\mathrm{T}=5.2$

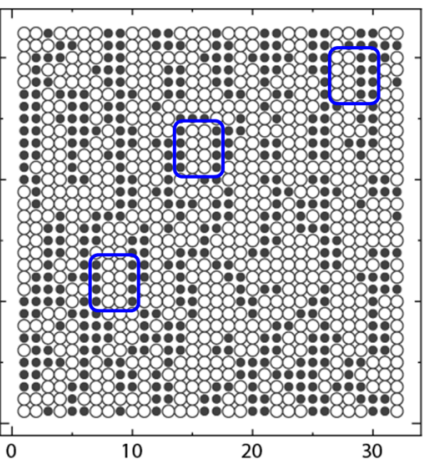

(d) $\quad \mathrm{T}=\mathbf{7 . 0}$

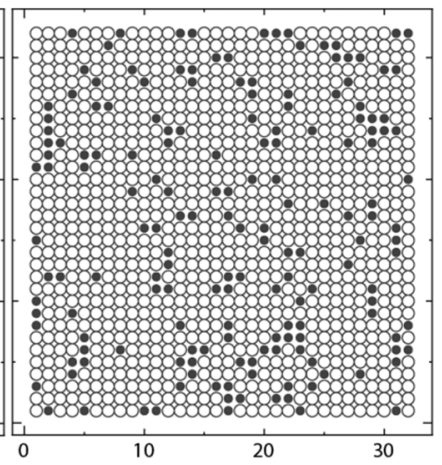

FIG. 13. Spatial configuration of HS (open circles) and LS (closed circles) states at typical temperatures. (a) $T=3.4$ (transition from IP to LS state), (b) $T=4.0$ (IP), (c) $T=5.2$, and (d) $T=7.0$ (HS state). The boxes at $T=5.2$ indicate the short-range HS-HS-LS-LS order. 

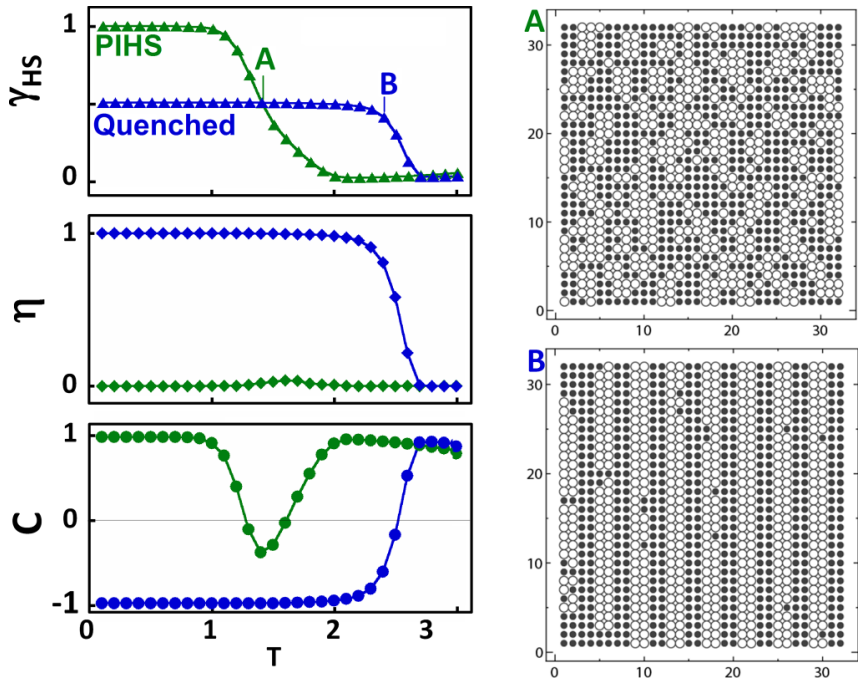

FIG. 14. Temperature variation during the warming process of the HS fraction $\gamma_{H S}$ (top), the order parameters $\eta$ (middle), and the correlation function $C$ (bottom) (MC simulations). The green line is the pathway from the PIHS state, and the blue line is the pathway from the quenched state. The spatial configuration of HS and LS states is shown at $T=1.4$ (point A) and $T=2.2$ (point B) on the PIHS and quenched pathways, respectively. For the calculations, we use the parameter set $\left(J_{\mathrm{A}} / J=1.6, J_{4} / J=0.2, D=8\right.$, and $\left.g=30\right)$. For the pathway starting from the PIHS state at $T=0.1$, we set $H=-D+T k_{\mathrm{B}} \ln (g) / 2=-2.4$ if $-D+T k_{\mathrm{B}} \ln (g) / 2<-2.4$ (see the text). For the process starting from the quenched state $(++$ - ) at $T=0.1$, we set $H=-D+T k_{\mathrm{B}} \ln (g) / 2=-3.0$ if $-D+$ $T k_{\mathrm{B}} \ln (g) / 2<-3.0$ (see the text). These calculations reproduce well the experimental curve shown in Fig. 6.

1. In experiments, $\gamma_{H S}$ is around 0.2 at low temperature, not 0.5 as found in our model. We understand that this reduction comes from the fact that the experimental cooling is not fast enough to completely quench the $(++--)$ structure. However, our calculation can satisfactorily reproduce the different relaxation pathways from PIHS and the quenched states found in the experiment. Although we need to adjust parameters to reproduce the whole temperature dependence of order parameters quantitatively, the qualitative feature is well explained within the model.

\section{CONCLUSION}

The molecular bistability of SCO systems and the frustration of molecular interactions at different ranges can give rise to different competing false ground states and ordered structures with defined sequence of spatially ordered molecules in different electronic states. We experimentally evidenced a two-step thermal conversion associated with a continuous phase transition from the HS state to an IP where only $50 \%$ of the molecules reach the LS state. In this IP, a long-range ordered HS-HS-LS-LS structure forms. Another discontinuous phase transition occurs at lower temperature towards the completely LS phase. In addition, we found experimentally that three competing states exist at low temperature: the LS state, the PIHS state, and the quenched IP. We used an ANNNI-like model, taking into account through a temperature dependent field the entropy driven nature of the molecular state conversion, and studied the evolution of the system by a $\mathrm{MF}$ approach and MC simulation. It allowed us to describe the phase diagram of such a system and reproduced the main experimental features mentioned above. The important point is that in the relaxation process from the PIHS state, the long-range ordered HS-HS-LS-LS structure cannot form in this system. Only a local HS-HS-LS-LS order forms during the relaxation from the PIHS state, similar to the one observed above the IP. Such local order of HS and LS states was also reported at thermal equilibrium for another SCO compound [40]. The present model can be used to explain ordering phenomena in various $\mathrm{Fe}^{\mathrm{II}}, \mathrm{Fe}^{\mathrm{III}}$ [20-38], and also Mn [63] SCO materials and can be extended to describe more complex ordering such as nanoscale self-hosting of HS and LS molecular states where two symmetry breaking order parameters are involved [21] as well as the formation of SSCW with periodicity incommensurate with the periodicity of the lattice [39]. In addition to the phenomenological ferro" and antiferro couplings introduced here, a more realistic coupling based on elastic interaction may be developed in the near future in the frame of the mechanoelastic model.

\section{ACKNOWLEDGMENTS}

This paper was supported by Fonds Européen de Développement Economique et Régional (FEDER, Project Matrics), the Institut Universitaire de France (E.C.), the National Research Agency (ANR-13-BS04-0002) the Région Bretagne (CREATE Ultimate 4146), Rennes Métropole, and University Rennes 1 (Post-Doc funding of H.W.). The present work was supported by Grants-in-Aid for Scientific Research C (25400391) from MEXT of Japan. The numerical calculations were supported by the supercomputer center of ISSP of Tokyo University.
[1] Spin-Crossover Materials: Properties and Applications, edited by M. Halcrow (John Wiley \& Sons, Ltd., West Sussex, 2013).

[2] A. Bousseksou, G. Molnar, L. Salmon, and W. Nicolazzi, Chem. Soc. Rev. 40, 3313 (2011).

[3] S. Decurtins, P. Gütlich, C. P. Köhler, H. Spiering, and A. Hauser, Chem. Phys. Lett. 105, 1 (1984).

[4] J. F. Létard, L. Capes, G. Chastanet, N. Moliner, S. Létard, J. A. Real, and O. Kahn, Chem. Phys. Lett. 313, 115 (1999).

[5] P. Chakraborty, A. Tissot, L. Peterhans, L. Guénée, C. Besnard, P. Pattison, and A. Hauser, Phys. Rev. B 87, 214306 (2013).
[6] M. Buron-Le Cointe, J. Hébert, C. Baldé, N. Moisan, L. Toupet, P. Guionneau, J. F. Létard, E. Freysz, H. Cailleau, and E. Collet, Phys. Rev. B. 85, 064114 (2012).

[7] S. Pillet, V. Legrand, M. Souhassou, and C. Lecomte, Phys. Rev. B 74, 140101 (2006).

[8] A. Goujon, F. Varret, K. Boukheddaden, C. Chong, J Jeftic, Y. Garcia, A. D. Naik, J. C. Ameline, and E. Collet, Inorg. Chim. Acta 361, 4055 (2008).

[9] C. Enachescu, A. Hauser, J. J. Girerd, and M. L. Boillot, ChemPhysChem 7, 1127 (2006). 
[10] M. Lorenc, J. Hébert, N. Moisan, E. Trzop, M. Servol, M. Buron-Le Cointe, H. Cailleau, M. L. Boillot, E. Pontecorvo, M. Wulff, S. Koshihara, and E. Collet, Phys. Rev. Lett. 103, 028301 (2009).

[11] S. Bonhommeau, G. Molnar, S. Cobo, D. Ostrovskii, and A. Bousseksou, Polyhedron 28, 1610 (2009).

[12] E. Collet, M. Lorenc, M. Cammarata, L. Guérin, M. Servol, A. Tissot, M.-L. Boillot, H. Cailleau, and M. Buron-Le Cointe, Chem. Eur. J. 18, 2051 (2012).

[13] M. Lorenc, Ch. Balde, W. Kaszub, A. Tissot, N. Moisan, M. Servol, M. Buron-Le Cointe, H. Cailleau, P. Chasle, P. Czarnecki, M. L. Boillot, and E. Collet, Phys. Rev. B 85, 054302 (2012).

[14] R. Bertoni, M. Lorenc, A. Tissot, M. Servol, M.-L. Boillot, and E. Collet, Angew. Chem. Int. Ed. 51, 7485 (2012).

[15] E. Collet, N. Moisan, C. Baldé, R. Bertoni, E. Trzop, C. Laulhé, M. Lorenc, M. Servol, H. Cailleau, A. Tissot, M.L. Boillot, T. Graber, R. Henning, P. Coppens, and M. Buron, Phys. Chem. Chem. Phys. 14, 6192 (2012).

[16] A. Marino, P. Chakraborty, M. Servol, M. Lorenc, E. Collet, and A. Hauser, Angew. Chem. Int. Ed. 53, 3863 (2014).

[17] M. Cammarata, R. Bertoni, M. Lorenc, H. Cailleau, S. Di Matteo, C. Mauriac, S. F. Matar, H. Lemke, M. Chollet, S. Ravy, C. Laulhé, J. F. Létard, and E. Collet, Phys. Rev. Lett. 113, 227402 (2014)

[18] A. Marino, M. Cammarata, S. F. Matar, J.-F. Létard, G. Chastanet, H. T. Lemke, and E. Collet, Struct. Dyn. 3, 023605 (2016).

[19] R. Bertoni, M. Lorenc, A. Tissot, M.-L. Boillot, and E. Collet, Coord. Chem. Rev. 282-283, 66 (2015).

[20] N. Bréfuel, H. Watanabe, L. Toupet, J. Come, M. Kojima, N. Matsumoto, E. Collet, K. Tanaka, and J. P. Tuchagues, Ang. Chem. Int. Ed 48, 9304 (2009).

[21] N. Bréfuel, E. Collet, H. Watanabe, M. Kojima, N. Matsumoto, L. Toupet, K. Tanaka, and J. P. Tuchagues, Chem. Eur. J. 16, 14060 (2010).

[22] N. Moliner, A. B. Gaspar, M. C. Munoz, V. Niel, J. Cano, and J. A. Real, Inorg. Chem. 40, 3986 (2001).

[23] J. Klingele, D. Kaase, M. H. Klingele, J. Lach, and S. Demeshko, Dalton Trans. 39, 1689 (2010).

[24] G. S. Matouzenko, J.-F. Létard, S. Lecocq, A. Bousseksou, L. Capes, L. Salmon, M. Perrin, O. Kahn, and A. Collet, Eur. J. Inorg. Chem. 11, 2935 (2001).

[25] B. Li, R.-J. Wei, J. Tao, R.-B. Huang, L.-S. Zheng, and Z. Zheng, J. Am. Chem. Soc. 132, 1558 (2010).

[26] M. Yamada, M. Ooidemizu, Y. Ikuta, S. Osa, N. Matsumoto, S. Iijima, M. Kojima, F. Dahan, and J.-P. Tuchagues, Inorg. Chem. 42, 8406 (2003).

[27] S. Bonnet, G. Molnar, J. S. Costa, M. A. Siegler, A. L. Spek, A. Bousseksou, W.-T. Fu, P. Gamez, and J. Reedijk, Chem. Mater. 21, 1123 (2009)

[28] S. Bonnet, M. A. Siegler, J. S. Costa, G. Molnar, A. Bousseksou, A. L. Spek, P. Gamez, and J. Reedijk, Chem. Commun. 5619 (2008).

[29] V. A. Money, C. Carbonera, J. Elhaïk, M. A. Halcrow, J. A. K. Howard, and J.-F. Létard, Chem. Eur. J. 13, 5503 (2007).

[30] M. Nihei, H. Tahira, N. Takahashi, Y. Otake, Y. Yamamura, K. Saito, and H. Oshio, J. Am. Chem. Soc. 132, 3553 (2010).

[31] D. Chernyshov, M. Hostettler, K. W. Törnroos, and H.-B. Bürgi, Angew. Chem. Int. Ed. 42, 3825 (2003).
[32] C. F. Sheu, S. Pillet, Y.-C. Lin, S.-M. Chen, I.-J. Hsu, C. Lecomte, and Y. Wang, Inorg. Chem. 47, 10866 (2008).

[33] M. Yamada, H. Hagiwara, H. Torigoe, N. Matsumoto, M. Kojima, F. Dahan, J.-P. Tuchagues, N. Re, and S. Iijima, Chem. Eur. J. 12, 4536 (2006).

[34] M. Griffin, S. Shakespeare, H. J. Shepherd, C. J. Harding, J.-F. Létard, C. Desplanches, A. E. Goeta, J. A. K. Howard, A. K. Powell, V. Mereacre, Y. Garcia, A. D. Naik, H. MüllerBunz, and G. G. Morgan, Angew. Chem. Int. Ed. 50, 896 (2011).

[35] D. Boinnard, A. Bousseksou, A. Dworkin, J.-M. Savariault, F. Varret, and J. P. Tuchagues, Inorg. Chem. 33, 271 (1994).

[36] B. A. Leita, S. M. Neville, G. J. Halder, B. Moubaraki, C. J. Kepert, J.-F. Létard, and K. S. Murray, Inorg. Chem. 46, 8784 (2007).

[37] S. Pillet, E. E. Bendeif, S. Bonnet, H. J. Shepherd, and P. Guionneau, Phys. Rev. B 86, 064106 (2012).

[38] K. D. Murnaghan, C. Carbonera, L. Toupet, M. Griffin, M. M. Ditru, C. Desplanches, Y. Garcia, E. Collet, J. F. Létard, and G. Morgan, Chem. Eur. J. 20, 5613 (2014).

[39] E. Collet, H. Watanabe, N. Bréfuel, L. Palatinus, L. Roudaut, L. Toupet, K. Tanaka, J.-P. Tuchagues, P. Fertey, S. Ravy, B. Toudic, and H. Cailleau, Phys. Rev. Lett. 109, 257206 (2012).

[40] S. Neville, B. Leita, G. Halder, C. Kepert, B. Moubaraki, J.-F. Létard, and K. S. Murray, Chem. Eur. J. 14, 10123 (2008).

[41] A. Bousseksou, F. Varret, and J. Nasser, J. Phys. I France 3, 1463 (1993).

[42] D. Chernyshov, H.-B. Burgi, M. Hostettler, and K. W. Törnroos, Phys. Rev. B 70, 094116 (2004).

[43] M. Nishino, K. Boukheddaden, S. Miyashita, and F. Varret, Phys. Rev. B 68, 224402 (2003).

[44] K. Boukheddaden, J. Linares, R. Tanasa, and C. Chong, J. Phys.: Condens. Matter 19, 106201 (2007).

[45] L. Landau, Ukr. J. Phys. 53, 25 (2008).

[46] A. Marino, M. Buron-Le Cointe, M. Lorenc, L. Toupet, R. Henning, A. D. DiChiara, K. Moffat, N. Bréfuel, and E. Collet, Faraday Discuss. 177, 363 (2015).

[47] E. Collet, M. L. Boillot, J. Hebert, N. Moisan, M. Servol, M. Lorenc, L. Toupet, M. Buron-Le Cointe, A. Tissot, and J. Sainton, Acta Cryst. B 65, 474 (2009).

[48] L. Guérin, J. Hébert, M. Buron-Le Cointe, S. I. Adachi, S. Y. Koshihara, H. Cailleau, and E. Collet, Phys. Rev. Lett. 105, 246101 (2010).

[49] A. Bousseksou, A. J. Nasser, J. Linares, K. Boukheddaden, and F. Varret, J. Phys. I France 2, 1381 (1992).

[50] S. Miyashita, Y. Konishi, H. Tokoro, M. Nishino, K. Boukheddaden, and F. Varret, Prog. Theor. Phys. 114, 719 (2005).

[51] M. Nishino, K. Boukheddaden, Y. Konishi, and S. Miyashita, Phys. Rev. Lett. 98, 247203 (2007)

[52] S. Miyashita, Y. Konishi, M. Nishino, H. Tokoro, and P. A. Rikvold, Phys. Rev. B 77, 014105 (2008).

[53] C. Enachescu, M. Nishino, S. Miyashita, in Spin-Crossover Materials: Properties and Applications, edited by M. Halcrow (John Wiley \& Sons, Ltd., West Sussex, 2013), pp. 455-474.

[54] T. Nakada, T. Mori, S. Miyashita, M. Nishino, S. Todo, W. Nicolazzi, and P. A. Rikvold, Phys. Rev. B 85, 054408 (2012).

[55] J. A. Real, H. Bolvin, A. Bousseksou, A. Dworkin, O. Kahn, F. Varret, and J. Zarembowitch, J. Am. Chem. Soc. 114, 4650 (1992). 
[56] K. Boukheddaden, J. Linares, H. Spiering, and F. Varret, Eur. Phys. J. B 15, 317 (2000).

[57] K. Boukheddaden, J. Linares, E. Codjovi, F. Varret, V. Niel, and J. A. Real, J. Appl. Phys. 93, 1 (2003).

[58] M. Nishino and S. Miyashita, Phys. Rev. B 88, 014108 (2013) and references therein.

[59] S. Miyashita, Proceedings of the Japan Academy, Series B 86, 643 (2010), and reference therein.
[60] P. Bak and J. von Boehm, Phys. Rev. B 21, 5297 (1980).

[61] M. E. Fisher and W. Selke, Phys. Rev. Lett. 441502 (1980).

[62] T. Tayagaki and K. Tanaka, Phys. Rev. Lett. 86, 2886 (2001).

[63] A. J. Fitzpatrick, E. Trzop, H. Müller-Bunz, M. M. Dîrtu, Y. Garcia, E. Collet, and G. G. Morgan, Chem. Commun. 51, 17540 (2015). 